\title{
Closed-Loop Stability Analysis of Discrete-Time Negative Imaginary Systems
}

DOI:

10.1016/j.sysconle.2018.02.006

\section{Document Version}

Accepted author manuscript

Link to publication record in Manchester Research Explorer

\section{Citation for published version (APA):}

Chen, H-J., \& Lanzon, A. (2018). Closed-Loop Stability Analysis of Discrete-Time Negative Imaginary Systems. Systems and Control Letters, 114, 52-58. https://doi.org/10.1016/j.sysconle.2018.02.006

\section{Published in:}

Systems and Control Letters

\section{Citing this paper}

Please note that where the full-text provided on Manchester Research Explorer is the Author Accepted Manuscript or Proof version this may differ from the final Published version. If citing, it is advised that you check and use the publisher's definitive version.

\section{General rights}

Copyright and moral rights for the publications made accessible in the Research Explorer are retained by the authors and/or other copyright owners and it is a condition of accessing publications that users recognise and abide by the legal requirements associated with these rights.

\section{Takedown policy}

If you believe that this document breaches copyright please refer to the University of Manchester's Takedown Procedures [http://man.ac.uk/04Y6Bo] or contact uml.scholarlycommunications@manchester.ac.uk providing relevant details, so we can investigate your claim.

\section{OPEN ACCESS}




\title{
Closed-Loop Stability Analysis of Discrete-Time

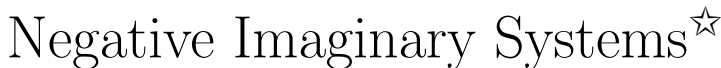

\author{
Hsueh-Ju Chen*, Alexander Lanzon \\ School of Electrical and Electronic Engineering, University of Manchester, Sackville \\ Street, Manchester M13 9PL, U.K.
}

\begin{abstract}
We derive necessary and sufficient conditions for stability analysis of a positive feedback interconnection of a discrete-time negative imaginary system and a discrete-time strictly negative imaginary system. General stability analysis results for continuous-time negative imaginary systems connected in positive feedback have recently been proposed. Those recent results extend previous theorems by removing restrictive assumptions on the infinite frequency gains imposed in the earlier literature and by extending the class of negative imaginary systems for which the results are applicable to include systems with free body dynamics (i.e., poles at the origin). Here, we present the discrete-time counterparts of the aforementioned recently developed results which specialise to simple and easy-to-check conditions under specific assumptions. Last, we illustrate some of the results by several examples.
\end{abstract}

Keywords: Discrete-time; negative imaginary systems; feedback stability; robust control.

\section{Introduction}

Negative imaginary systems theory is emerging as a powerful complement to positive real theory and passivity theory. The negative imaginary

\footnotetext{
This work was supported by the Engineering and Physical Sciences Research Council (EPSRC) [grant number EP/R008876/1]. All research data supporting this publication are directly available within this publication.

* Corresponding author.

Email addresses: hsueh-ju.chen@manchester.ac.uk (Hsueh-Ju Chen), Alexander.Lanzon@manchester.ac.uk (Alexander Lanzon)
} 
systems class was first studied in [1]. Negative imaginary systems arise in a wide variety of applications, including nano-positioning systems $[2,3,4,5]$, multi-agent systems [6, 7], lightly damped structure $[8,9,10]$, vehicle platoons [11], etc. A rich sequence of results have also appeared in the theory of negative imaginary systems in recent years, including extensions to Hamiltonian systems [12], non-rational systems $[13,14,15,16]$, non-proper systems [13, 17], infinite-dimensional systems [18], descriptor systems [19], strongly strict negative-imaginary systems [20] and controller synthesis for negative imaginary systems $[21,22,23,24]$. According to $[25,26]$, some possible future work for filtering problems could be further developed.

Stability analysis results of positive feedback interconnections of negative imaginary systems play a central role in negative imaginary systems theory. [1] proposed that, under assumptions on the gains of systems at infinite frequency, a necessary and sufficient condition for the internal stability of a positive feedback interconnection of negative imaginary systems can be expressed as a one-sided restriction on the dc loop gain. This stability result was shown to hold true even for negative imaginary systems with poles on the imaginary axis [27]. These key results have subsequently been developed further to allow negative imaginary systems to have possible poles at the origin [28]. [29] then sought to remove the assumptions on the infinite frequency gains, i.e., $M(\infty) N(\infty)=0$ and $N(\infty) \geqslant 0$, by using integral quadratic constraint theory and derived sufficient conditions (which are not necessary) for stability analysis. Necessary and sufficient conditions that remove the assumptions on the infinity frequency gains were recently derived in [30]. In contrast with complicated matrix factorisations used in [28] which loose intuition and restrict the applicability of the results and in contrast with sufficiently only conditions developed in [29], a linear shift transformation technique is used in [30] to establish general necessary and sufficient stability analysis results applicable for the full class of negative imaginary systems including those with free body dynamics (i.e., poles at the origin).

The above theory has all been developed in continuous-time. The notion of a discrete-time negative imaginary systems was proposed in $[14,31]$ to fill the gap in the literature. By using a bilinear transformation, a discrete-time negative imaginary lemma was derived, in terms of a discrete-time state-space representation, to characterise discrete-time negative imaginary systems $[14$, 31]. Furthermore, it was shown in [14] that the stability of discrete-time negative imaginary systems only depends on gains at $z=+1$ under specific assumptions analogous to the early assumptions in continuous-time. Here, 
we extend the stability theorem proposed in [14] for the full class of real, rational, proper discrete-time negative imaginary systems available in the literature without imposing the restrictive assumptions, i.e., $P(-1) Q(-1)=$ 0 and $Q(-1) \geqslant 0$. The results in this paper can be considered as, not only generalisations of previous work $[14,31]$ but also, discrete-time counterparts of the general continuous-time results in [30].

In this paper, we first state the definitions of discrete-time negative imaginary systems. We then remove two restrictive assumptions in the existing literature, i.e., $P(-1) Q(-1)=0$ and $Q(-1) \geqslant 0$, imposed in [14] and subsequently derive necessary and sufficient conditions for internal stability of a discrete-time negative imaginary system without poles at $z=+1$ and $z=-1$ connected in positive feedback with a discrete-time strictly negative imaginary system. Then, these results are extended to the case where a discrete-time negative imaginary system with possible poles at $z=+1$ is connected in positive feedback with a discrete-time strictly negative imaginary system. Furthermore, we specialise these general stability theorems in the single-input single-output (SISO) setting to reveal simple and intuitive tests. Additional multiple-input multiple-output (MIMO) specialisations are also given as corollaries to give simple and elegant tests for checking feedback stability. Stability conditions with or without a loop-shifting matrix $\Psi$ are also presented for determining the internal stability of discrete-time negative imaginary systems connected in positive feedback. Lastly, two examples are given to illustrate the importance of some of the results.

Notation: $\mathfrak{R}(a)$ represents the real part of a complex number $a . \bar{\lambda}(A)$ [respectively, $\underline{\lambda}(A)$ ] denote the largest [respectively, smallest] eigenvalue of a square complex matrix $A$ that has only real eigenvalues. $A^{*}$ and $A^{T}$ denote the complex conjugate transpose and transpose of a complex matrix $A$ respectively. $[P(z), Q(z)]$ denotes the positive feedback interconnection of $P(z)$ and $Q(z)$. $I_{m}$ denotes an identity matrix with dimensions $m$ by $m$.

\section{Preliminaries}

We first recall the notion of a discrete-time negative imaginary system with possible poles at $z=+1$.

Definition 1. ([14, 31]). Let $R(z)$ be a discrete-time, real, rational, proper transfer function. Then, $R(z)$ is said to be Discrete-time Negative Imaginary (D-NI) if 
1) $R(z)$ has no poles in $\{z \in \mathbb{C}:|z|>1\}$;

2) $j\left[R\left(e^{j \theta}\right)-R\left(e^{j \theta}\right)^{*}\right] \geqslant 0$ for all $\theta \in(0, \pi)$ except the values of $\theta$ where $z=e^{j \theta}$ is a pole of $R(z)$;

3) if $z_{0}=e^{j \theta_{0}}$ with $\theta_{0} \in(0, \pi)$ is a pole of $R(z)$, then it is a simple pole and the residue matrix $K_{0}=z_{0}^{-1} \lim _{z \rightarrow z_{0}}\left(z-z_{0}\right) j R(z)$ is Hermitian and positive semidefinite;

4) if $z=1$ is a pole of $R(z)$, then $\lim _{z \rightarrow 1}(z-1)^{k} R(z)=0$ for all integer $k \geqslant 3$ and $\lim _{z \rightarrow 1}(z-1)^{2} R(z)$ is Hermitian and positive semidefinite;

5) if $z=-1$ is a pole of $R(z)$, then $\lim _{z \rightarrow-1}(z+1)^{k} R(z)=0$ for all integer $k \geqslant 3$ and $\lim _{z \rightarrow-1}(z+1)^{2} R(z)$ is Hermitian and negative semidefinite.

[14] considers non-rational systems. To handle possibly non-rational systems, [14] impose a symmetric assumption. As stated in Remark 3.2 of [14], when one restricts attention to rational systems (as we do in this paper), the symmetric assumption is no longer needed. The five conditions in Lemma 3.2 of [14] with the condition corresponding to symmetry removed, are hence used to directly define rational discrete-time systems as in Definition 1 above. This definition is also identical to that used in [31].

The following definition describes discrete-time strictly negative imaginary systems.

Definition 2. ([14]). Let $R(z)$ be a discrete-time, real, rational, proper transfer function. Then, $R(z)$ is said to be Discrete-time Strictly Negative Imaginary (D-SNI) if

1) $R(z)$ has no poles in $\{z \in \mathbb{C}:|z| \geqslant 1\}$;

2) $j\left[R\left(e^{j \theta}\right)-R\left(e^{j \theta}\right)^{*}\right]>0$ for all $\theta \in(0, \pi)$.

\section{Main results, part 1: No poles at +1 and -1}

In [30], necessary and sufficient conditions for checking the internal stability of a positive feedback interconnection of a continuous-time, proper, negative imaginary system without poles at the origin and a continuous-time strictly negative imaginary system were derived. The necessary and sufficient 


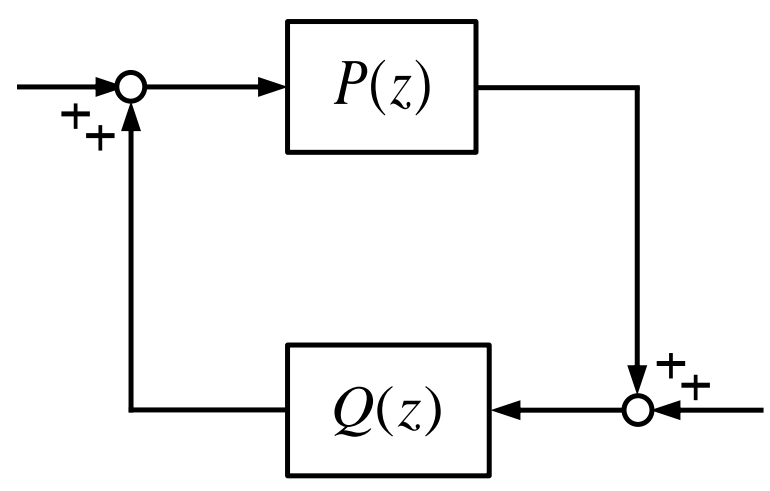

Figure 1: Positive feedback interconnection of $P(z)$ and $Q(z)$.

conditions in [30] generalised the original result in [1] by removing restrictive assumptions on the infinite frequency gains of the two systems. In this section, we consider the case where a discrete-time negative imaginary system and a discrete-time strictly negative imaginary system are interconnected via positive feedback as shown in Figure 1. We hence introduce discrete-time feedback stability theorems that remove restrictive assumptions imposed in earlier literature (e.g., [14]). These results are applicable for negative imaginary systems without poles at $z=+1$ and $z=-1$ and they are hence discrete-time counterparts of the work in Section 3 of [30].

Theorem 3. Let $P(z)$ be a discrete-time, real, rational, proper, negative imaginary system without poles at $z=+1$ and $z=-1$, and let $Q(z)$ be a discrete-time, real, rational, proper, strictly negative imaginary system. Then, $[P(z), Q(z)]$ is internally stable if and only if

$$
\begin{aligned}
& I-P(-1) Q(-1) \text { is nonsingular, } \\
& \bar{\lambda}\left[[I-P(-1) Q(-1)]^{-1}(P(-1) Q(1)-I)\right]<0, \text { and } \\
& \bar{\lambda}\left[[I-Q(1) P(-1)]^{-1}(Q(1) P(1)-I)\right]<0 .
\end{aligned}
$$

Proof. Let $M(s)=P((1+s) /(1-s))$ and $N(s)=Q((1+s) /(1-s))$ via the bilinear transformation $z=(1+s) /(1-s)$. Then, the result follows from [30, Theorem 9].

Note that the inequality conditions in Theorem 3 (and indeed in all negative imaginary results) are one-sided restrictions because the maximum eigenvalue, of matrices that have only real eigenvalues, can be either positive or 
negative.

Also, note that no poles at $z=+1$ in discrete-time corresponds to no poles at the origin in continuous-time, whereas no poles at $z=-1$ in discretetime corresponds to no poles at infinite frequency (i.e., a proper system) in continuous-time.

Theorem 3 removes the assumptions imposed in [14, Theorem 4.1], i.e., $Q(-1) \geqslant 0$ and $P(-1) Q(-1)=0$, and as a consequence generalises that result.

The following example is used to demonstrate the usefulness of the result stated in Theorem 3.

Example 1. Consider a positive feedback interconnection of $P(z)$ and $Q(z)$ as shown in Figure 1 where $P(z)=\left[\begin{array}{cc}\frac{-z+1}{6 z+4} & \frac{-5 z-5}{6 z+4} \\ \frac{-9 z^{2}-10 z-1}{12 z^{2}+8 z} & \frac{15 z^{2}+32 z+13}{12 z^{2}+8 z}\end{array}\right]$ and $Q(z)=$ $\left[\begin{array}{cc}\frac{5 z+5}{6 z+4} & \frac{-17 z-13}{6 z+4} \\ \frac{-33 z^{2}-26 z-1}{12 z^{2}+8 z} & \frac{15 z^{2}+32 z+13}{12 z^{2}+8 z}\end{array}\right]$. Both $P(z)$ and $Q(z)$ are discrete-time, strictly negative imaginary systems. Since $I-P(-1) Q(-1)=\left[\begin{array}{cc}1 & -2 \\ -2 & 0\end{array}\right]$ is nonsingular, the feedback system is well-posed. The internal stability of the closed-loop system cannot be determined via [14, Theorem 4.1] because $P(-1) Q(-1)=\left[\begin{array}{ll}0 & 2 \\ 2 & 1\end{array}\right] \neq 0$ and $Q(-1)=\left[\begin{array}{cc}0 & -2 \\ -2 & -1\end{array}\right] \neq 0$. Using the stability conditions in Theorem 3, we obtain $\bar{\lambda}\left[[I-P(-1) Q(-1)]^{-1}(P(-1) Q(1)-\right.$ $I)]=-0.1340<0$ and $\bar{\lambda}\left[[I-Q(1) P(-1)]^{-1}(Q(1) P(1)-I)\right]=8 \nless 0$. Hence, the positive feedback interconnection of $P(z)$ and $Q(z)$ is not internally stable. Note that the fact that the closed-loop system is not internally stable can be confirmed by checking the poles of $(I-P Q)^{-1}$. Since two of the poles of $(I-P Q)^{-1}$ are at $z=6.3702$ and $z=-1.4981$, which are not inside the unit circle, the feedback system is not internally stable.

When we impose identical assumptions as in [14, Theorem 4.1], i.e., $P(-1) Q(-1)=$ 0 and $Q(-1) \geqslant 0$, the stability conditions in Theorem 3 reduce to the same result as that in $[14$, Theorem 4.1$]$.

Corollary 4. Let $P(z)$ be a discrete-time, real, rational, proper, negative imaginary system without poles at $z=+1$ and $z=-1$, and let $Q(z)$ be a 
discrete-time, real, rational, proper, strictly negative imaginary system. Let $P(-1) Q(-1)=0$ and $Q(-1) \geqslant 0$. Then, $[P(z), Q(z)]$ is internally stable if and only if $\bar{\lambda}[P(1) Q(1)]<1$.

Proof. This is a direct consequence of Theorem 3 because

1) $P(-1) Q(-1)=0 \Rightarrow I-P(-1) Q(-1)$ is nonsingular;

2) $\bar{\lambda}\left[[I-P(-1) Q(-1)]^{-1}(P(-1) Q(1)-I)\right]<0$

$\Leftrightarrow \bar{\lambda}[P(-1) Q(1)]<1$

$\Leftrightarrow Q(1)^{1 / 2} P(-1) Q(1)^{1 / 2}<I$

(since $Q(1)>Q(-1) \geqslant 0$ via [14, Lemma 3.9])

$\Leftrightarrow P(-1)<Q(1)^{-1}$;

3) $\bar{\lambda}\left[[I-Q(1) P(-1)]^{-1}(Q(1) P(1)-I)\right]<0$

$\Leftrightarrow \bar{\lambda}\left[\left[Q(1)^{-1}-P(-1)\right]^{-1}\left(P(1)-Q(1)^{-1}\right)\right]<0$

(since $Q(1)>Q(-1) \geqslant 0$ via $[14$, Lemma 3.9])

$\Leftrightarrow\left[Q(1)^{-1}-P(-1)\right]^{-1 / 2}\left(P(1)-Q(1)^{-1}\right)\left[Q(1)^{-1}-P(-1)\right]^{-1 / 2}<0$

(since $Q(1)^{-1}>P(-1)$ via above)

$\Leftrightarrow P(1)-Q(1)^{-1}<0$

$\Leftrightarrow \bar{\lambda}[P(1) Q(1)]<1$.

But $\bar{\lambda}[P(1) Q(1)]<1 \Leftrightarrow P(1)<Q(1)^{-1} \Rightarrow P(-1)<Q(1)^{-1}$ (since $P(-1) \leqslant$ $P(1)$ via $[14$, Lemma 3.9]). This concludes the proof.

The following corollary applies to a negative imaginary system $P(z)$ with a blocking zero at $z=-1$. The stability condition is the same as in Corollary 4 which is a very simple necessary and sufficient condition for internal stability.

Corollary 5. Let $P(z)$ be a discrete-time, real, rational, proper, negative imaginary system without poles at $z=+1$ and $z=-1$, and let $Q(z)$ be a discrete-time, real, rational, proper, strictly negative imaginary system. Let $P(-1)=0$. Then, $[P(z), Q(z)]$ is internally stable if and only if $\bar{\lambda}[P(1) Q(1)]<1$.

Proof. Direct simplification of Theorem 3 on using $P(-1)=0$. 
As shown in [30, Theorem 14], other equivalent feedback stability conditions can also be obtained. The following result is a discrete-time counterpart of [30, Theorem 14].

Theorem 6. Let $P(z)$ be a discrete-time, real, rational, proper, negative imaginary system without poles at $z=+1$ and $z=-1$, and let $Q(z)$ be a discrete-time, real, rational, proper, strictly negative imaginary system. Then, $[P(z), Q(z)]$ is internally stable if and only if

$$
\begin{aligned}
& I-P(-1) Q(-1) \text { is nonsingular, } \\
& \bar{\lambda}\left[(P(1) Q(-1)-I)[I-P(-1) Q(-1)]^{-1}\right]<0, \text { and } \\
& \bar{\lambda}\left[(Q(1) P(1)-I)[I-Q(-1) P(1)]^{-1}\right]<0 .
\end{aligned}
$$

Proof. Let $M(s)=P((1+s) /(1-s))$ and $N(s)=Q((1+s) /(1-s))$ via the bilinear transformation $z=(1+s) /(1-s)$. Then, the result follows from [30, Theorem 14].

We can, of course, use Theorem 6 instead of Theorem 3 to show the internal stability of a feedback system. Although the following example is taken directly from Example 1 to show that the conditions in Theorem 6 can be used equivalently instead of the conditions in Theorem 3, this example also illustrates that each of the individual inequalities in the conditions of Theorem 6 are not separately equivalent to either individual inequality in the conditions of Theorem 3.

Example 2. Consider a positive feedback interconnection of $P(z)$ and $Q(z)$ as shown in Figure 1 where $P(z)=\left[\begin{array}{cc}\frac{-z+1}{6 z+4} & \frac{-5 z-5}{6 z+4} \\ \frac{-9 z^{2}-10 z-1}{12 z^{2}+8 z} & \frac{15 z^{2}+32 z+13}{12 z^{2}+8 z}\end{array}\right]$ and $Q(z)=$ $\left[\begin{array}{cc}\frac{5 z+5}{6 z+4} & \frac{-17 z-13}{6 z+4} \\ \frac{-33 z^{2}-26 z-1}{12 z^{2}+8 z} & \frac{15 z^{2}+32 z+13}{12 z^{2}+8 z}\end{array}\right]$. Both $P(z)$ and $Q(z)$ are discrete-time strictly negative imaginary systems. The closed-loop system is well-posed as shown in Example 1. Instead of applying the conditions in Theorem 3, via Theorem 6 we have $\bar{\lambda}\left[(P(1) Q(-1)-I)[I-P(-1) Q(-1)]^{-1}\right]=3.3028 \nless 0$ and $\bar{\lambda}\left[(Q(1) P(1)-I)[I-Q(-1) P(1)]^{-1}\right]=1.5447 \nless 0$. Hence, the positive feedback interconnection between $P(z)$ and $Q(z)$ is not internally stable also via Theorem 6 . Note that both inequalities are violated in the conditions of Theorem 6 whereas only one inequality was violated in the conditions of Theorem 3 in this specific example illustrating the fact that each inequality 
in the conditions of Theorem 6 is not a simple re-write of each inequality in the conditions in Theorem 3.

Different assumptions can be imposed to simplify the stability result in Theorem 6 . Under the assumption $P(1)>0$, the loop gain condition at $z=+1$ can again be used to determine the internal stability of the closed-loop system, but unlike Corollary 4 , there is no sign restriction on $Q(-1)$.

Corollary 7. Let $P(z)$ be a discrete-time, real, rational, proper, negative imaginary system without poles at $z=+1$ and $z=-1$, and let $Q(z)$ be a discrete-time, real, rational, proper, strictly negative imaginary system. Let $P(-1) Q(-1)=0$ and $P(1)>0$. Then, $[P(z), Q(z)]$ is internally stable if and only if $\bar{\lambda}[P(1) Q(1)]<1$.

Proof. This is a direct consequence of Theorem 6 because

1) $P(-1) Q(-1)=0$ implies $I-P(-1) Q(-1)$ is nonsingular;

2) $\bar{\lambda}\left[(P(1) Q(-1)-I)[I-P(-1) Q(-1)]^{-1}\right]<0$

$$
\begin{aligned}
& \Leftrightarrow \bar{\lambda}[P(1) Q(-1)]<1 \\
& \Leftrightarrow P(1)^{1 / 2} Q(-1) P(1)^{1 / 2}<I \\
& \Leftrightarrow Q(-1)<P(1)^{-1} ;
\end{aligned}
$$

3) $\bar{\lambda}\left[(Q(1) P(1)-I)[I-Q(-1) P(1)]^{-1}\right]<0$

$$
\begin{aligned}
\Leftrightarrow & \bar{\lambda}\left[\left(Q(1)-P(1)^{-1}\right)\left[P(1)^{-1}-Q(-1)\right]^{-1}\right]<0 \\
\Leftrightarrow & \bar{\lambda}\left[\left[P(1)^{-1}-Q(-1)\right]^{-1 / 2}\left(Q(1)-P(1)^{-1}\right)\left[P(1)^{-1}-Q(-1)\right]^{-1 / 2}\right]<0 \\
& \left(\text { since } P(1)^{-1}>Q(-1) \text { via above }\right) \\
\Leftrightarrow & Q(1)-P(1)^{-1}<0 \\
\Leftrightarrow & \bar{\lambda}[P(1) Q(1)]<1 .
\end{aligned}
$$

But $\bar{\lambda}[P(1) Q(1)]<1 \Leftrightarrow Q(1)<P(1)^{-1} \Rightarrow Q(-1)<P(1)^{-1}($ since $Q(-1)<$ $Q(1)$ via $[14$, Lemma 3.9]). This concludes the proof. 


\section{SISO specialisation}

We have presented feedback stability results for interconnected discretetime, negative imaginary systems without poles at $z=+1$ and $z=-1$ in Theorem 3 and Theorem 6. In the SISO case, these results can be further specialised, as shown in the following theorem, which is the discrete-time counterpart of [30, Theorem 17]. This specialisation is useful because it sheds some light as to why the stability conditions in Theorems 3 and 6 require a mixture of frequencies at $z=+1$ and $z=-1$. The SISO specialisation also allows a pictorial Nyquist interpretation of the results.

Lemma 8. Let $P(z)$ be a discrete-time, real, rational, proper, scalar negative imaginary system without poles at $z=+1$ and $z=-1$, and let $Q(z)$ be a discrete-time, real, rational, proper, scalar strictly negative imaginary system. Then, the following three statements are equivalent:

1) $[P(z), Q(z)]$ is internally stable;

2) either condition a) or condition b) holds:

a) $P(1) Q(1)<1, P(-1) Q(-1)<1$ and $P(-1) Q(1)<1$;

b) $P(1) Q(1)>1, P(-1) Q(-1)>1$ and $P(-1) Q(1)>1$;

3) either condition a) or condition b) holds:

a) $P(1) Q(1)<1, P(-1) Q(-1)<1$ and $P(1) Q(-1)<1$;

b) $P(1) Q(1)>1, P(-1) Q(-1)>1$ and $P(1) Q(-1)>1$.

Proof. Via Theorem 3, $[P(z), Q(z)]$ is internally stable if and only if $P(-1) Q(-1) \neq$ $1,(1-P(-1) Q(1)) /(P(-1) Q(-1)-1)<0$, and $(P(1) Q(1)-1) /(1-P(-1) Q(1))<$ 0 . These three conditions yield condition $2 \mathrm{a}$ ) or condition $2 \mathrm{~b})$.

Theorem 6 gives condition $3 \mathrm{a}$ ) or condition $3 \mathrm{~b}$ ).

The internal stability conditions in Lemma 8 can be further simplified as shown in Theorem 9.

Theorem 9. Let $P(z)$ be a discrete-time, real, rational, proper, scalar negative imaginary system without poles at $z=+1$ and $z=-1$, and let $Q(z)$ be a discrete-time, real, rational, proper, scalar strictly negative imaginary system. Then, $[P(z), Q(z)]$ is internally stable if and only if one of the following three mutually exclusive conditions holds: 
i) $P(1) Q(1)<1$ and $P(-1) Q(-1)<1$;

ii) $Q(-1)>0$ and $P(-1) Q(-1)>1$;

iii) $Q(1)<0$ and $P(1) Q(1)>1$.

Proof. Both conditions 2a) and 3a) in Lemma 8 reduce to condition i) in this theorem statement. This then implies that conditions $2 \mathrm{~b}$ ) and $3 \mathrm{~b}$ ) in Lemma 8 are equivalent.

Next recall that $Q(1)>Q(-1)$ and $P(1) \geqslant P(-1)$ via [14, Lemma 3.9]. Condition ii) and these two negative imaginary properties imply $Q(1)>$ $Q(-1)>0$ and $P(1) \geqslant P(-1)>0$. Then $P(1) Q(1)>P(1) Q(-1), P(1) Q(1) \geqslant$ $P(-1) Q(1), P(-1) Q(1)>P(-1) Q(-1)>1$ and $P(1) Q(-1) \geqslant P(-1) Q(-1)>$ 1. These inequalities then imply conditions $2 \mathrm{~b})$ and $3 \mathrm{~b})$ in Lemma 8. Similarly, condition iii) and the same two negative imaginary properties imply $0>Q(1)>Q(-1)$ and $0>P(1) \geqslant P(-1)$. Then $1<P(1) Q(1)<$ $P(1) Q(-1), 1<P(1) Q(1) \leqslant P(-1) Q(1), P(-1) Q(1)<P(-1) Q(-1)$ and $P(1) Q(-1) \leqslant P(-1) Q(-1)$. These inequalities then imply conditions $2 \mathrm{~b})$ and $3 \mathrm{~b})$ in Lemma 8.

To show the converse, consider the following five complimentary cases: $0<Q(-1)<Q(1), 0=Q(-1)<Q(1), Q(-1)<0<Q(1), Q(-1)<$ $Q(1)=0$, and $Q(-1)<Q(1)<0$. The three middle cases violate conditions $2 \mathrm{~b})$ and $3 \mathrm{~b}$ ) in Lemma 8 . Hence only two valid complimentary cases are permitted by conditions $2 \mathrm{~b}$ ) and $3 \mathrm{~b}$ ) in Lemma 8: $0<Q(-1)$ and $Q(1)<0$. Hence, condition 2b) (respectively, condition 3b) ) in Lemma 8 implies either condition ii) or condition iii) of this theorem statement.

The following example illustrates use of Theorem 9.

Example 3. Consider a positive feedback interconnection of $P(z)$ and $Q(z)$ as shown in Figure 1 where $P(z)=(z+2) / z$ and $Q(z)=(3-z) /(2 z)$. Both $P(z)$ and $Q(z)$ are strictly negative imaginary systems. Using Theorem 9 we conclude that the feedback interconnection of $P(z)$ and $Q(z)$ is not internally stable since $P(1) Q(1)=(3)(1)>1$ and $P(-1) Q(-1)=(-1)(-2)>1$, but $Q(1)=1 \nless 0$ and $Q(-1)=-2 \ngtr 0$. The fact that the closed-loop system is not internally stable can be confirmed by checking the poles of $(I-P Q)^{-1}$. Since these poles are at $z=1.5907$ and $z=-1.2573$, which are not inside the unit circle, the feedback system is not internally stable. 


\section{Main results, part 2: Allowing poles at +1}

In the previous section, we assumed that each of the discrete-time negative imaginary system did not have poles at $z=+1$ and $z=-1$ to obtain elegant and simple results which specialise to earlier results in the literature when identical assumptions as in the literature are additionally imposed. Here, we build on the results of Section 3 to give general stability theorems that allow discrete-time negative imaginary systems to have possible poles at $z=+1$. Note that while the restriction of no poles at $z=-1$ is natural in a discretetime setting because it corresponds to proper continuous-time systems via the bilinear transformation $z=(1+s) /(1-s)$, the restriction of no poles at $z=+1$ was unnatural and hence it will be removed here. Additionally, we show that the rather complex results stated in this section can be specialised to the simpler results of Section 3 under the same assumptions.

\subsection{Generalised Internal Stability Results for Discrete-Time Negative Imag-} inary Systems with Possible Poles at $z=+1$

In Section 3, we proposed feedback stability results which are only suitable for discrete-time, negative imaginary systems without poles at $z=+1$ and $z=-1$. In this subsection, we generalise those earlier results and propose general feedback stability theorems for real, rational, proper, discrete-time, negative imaginary systems with possible poles at $z=+1$.

Theorem 10. Let $P(z)$ be a discrete-time, real, rational, proper, negative imaginary system without poles at $z=-1$, and let $Q(z)$ be a discrete-time, real, rational, proper, strictly negative imaginary system. Let $\Psi<0$ be such that $\bar{\lambda}[P(-1) \Psi]<1$. Then, $[P(z), Q(z)]$ is internally stable if and only if

$$
\begin{aligned}
& I-P(-1) Q(-1) \text { is nonsingular, } \\
& \bar{\lambda}\left[[I-P(-1) Q(-1)]^{-1}[P(-1) Q(1)-I]\right]<0, \text { and } \\
& \bar{\lambda}\left[\lim _{z \rightarrow 1}\left[[I-\Psi P(-1)][I-Q(1) P(-1)]^{-1}[Q(1) P(z)-I][I-\Psi P(z)]^{-1}\right]\right]< \\
& 0 .
\end{aligned}
$$

Proof. Let $M(s)=P((1+s) /(1-s))$ and $N(s)=Q((1+s) /(1-s))$ via the bilinear transformation $z=(1+s) /(1-s)$. Then, the result follows from [30, Theorem 24].

We can also build on Theorem 6, instead of building on Theorem 3, and give another equivalent general stability theorem. 
Theorem 11. Let $P(z)$ be a discrete-time, real, rational, proper, negative imaginary system without poles at $z=-1$, and let $Q(z)$ be a discrete-time, real, rational, proper, strictly negative imaginary system. Let $\Psi<0$ be such that $\bar{\lambda}[P(-1) \Psi]<1$. Then, $[P(z), Q(z)]$ is internally stable if and only if

$I-P(-1) Q(-1)$ is nonsingular,

$$
\begin{aligned}
& \bar{\lambda}\left[\lim _{z \rightarrow 1}\left[[I-P(z) \Psi]^{-1}[P(z) Q(-1)-I][I-P(-1) Q(-1)]^{-1}[I-P(-1) \Psi]\right]\right]< \\
& 0, \text { and } \\
& \bar{\lambda}\left[\lim _{z \rightarrow 1}\left[[Q(1) P(z)-I][I-Q(-1) P(z)]^{-1}\right]\right]<0 .
\end{aligned}
$$

Proof. Let $M(s)=P((1+s) /(1-s))$ and $N(s)=Q((1+s) /(1-s))$ via the bilinear transformation $z=(1+s) /(1-s)$. Then, the result follows from [30, Theorem 26].

Remark 12. It can be easily shown that all the limits in the stability conditions are finite by [30, Lemma 20], [30, Remark 25], and [30, Remark 29] together with the bilinear transformation $z=(1+s) /(1-s)$. Additionally, the general stability conditions obtained in Theorem 10 and Theorem 11 depend on a matrix $\Psi$ fulfilling the properties $\Psi<0$ and $\bar{\lambda}[P(-1) \Psi]<1$. Note that $P(-1)$ is symmetric because of condition 2$)$ in Definition 1 together with a continuity and a limiting argument at $\theta=\pi$. Here $P(-1)$ has only real eigenvalues. Then, to construct $\Psi<0$ satisfying $\bar{\lambda}[P(-1) \Psi]<1$, it suffices to choose $\Psi=\varepsilon I$, where $\varepsilon$ is any strictly negative real number if $\underline{\lambda}(P(-1)) \geqslant 0$ and $\varepsilon$ is any real number in the interval $(1 / \underline{\lambda}(P(-1)), 0)$ if $\underline{\lambda}(P(-1))<0$. Finally, note that although there are many matrices $\Psi<0$ satisfying $\bar{\lambda}[P(-1) \Psi]<1$, only one arbitrary $\Psi<0$ satisfying $\bar{\lambda}[P(-1) \Psi]<1$ needs to be tested in Theorem 10 and Theorem 11 to conclude the internal stability of the feedback interconnection. This observation can be proven by using the bilinear transformation $z=(1+s) /(1-s)$ on [30, Theorem 35] (or [30, Theorem 36]).

\subsection{Specialisations of the Discrete-Time Generalised Internal Stability Con- ditions}

In the SISO case, if we assume that $P(z)$ has one or two poles at $z=+1$ but no poles at $z=-1$, the general stability results obtained in the previous subsection can be simplified to conditions that are easy to check. Note that the stability conditions in Corollary 13 do not involve the matrix $\Psi$. 
Corollary 13. Let $P(z)$ be a discrete-time, real, rational, proper, scalar, negative imaginary system without poles at $z=-1$ and $Q(z)$ be a discretetime, real, rational, proper, scalar, strictly negative imaginary system. Let $z=+1$ be a (single or double) pole of $P(z)$. Then, $[P(z), Q(z)]$ is internally stable if and only if one of the following two mutually exclusive conditions holds:

i) $P(-1) Q(-1)<1$ and $Q(1)<0$;

ii) $P(-1) Q(-1)>1$ and $Q(-1)>0$.

Proof. Writing $P(z)$ as a Laurent series, the three conditions in Theorem 11 simplify, in the scalar case, to $P(-1) Q(-1) \neq 1, Q(-1) /(1-P(-1) Q(-1))$ and $Q(1) / Q(-1)>0$ after taking the limit as $z \rightarrow 1$. It is easy to see that these three conditions are equivalent to either condition i) or condition ii) in this corollary statement using the negative imaginary property $Q(1)>Q(-1)$ via [14, Lemma 3.9].

In the MIMO case, under the assumption that $P(z)$ has a blocking zero at $z=-1$, the results obtained previously specialise to the following corollary.

Corollary 14. Let $P(z)$ be a discrete-time, real, rational, proper, negative imaginary system without poles at $z=-1$ but with $P(-1)=0$. Let $Q(z)$ be a discrete-time, real, rational, proper, strictly negative imaginary system. Let $\Psi<0$. Then, the following three conditions are equivalent:

1) $[P(z), Q(z)]$ is internally stable;

2) $\bar{\lambda}\left[\lim _{z \rightarrow 1}\left[[Q(1) P(z)-I][I-\Psi P(z)]^{-1}\right]\right]<0$;

3) $\bar{\lambda}\left[\lim _{z \rightarrow 1}\left[[I-P(z) \Psi]^{-1}[P(z) Q(-1)-I]\right]\right]<0$ and

$$
\bar{\lambda}\left[\lim _{z \rightarrow 1}\left[[Q(1) P(z)-I][I-Q(-1) P(z)]^{-1}\right]\right]<0 .
$$

Proof. Direct consequence from Theorem 10 and Theorem 11 on using $P(-1)=0$.

The next result, which is again valid in the MIMO case, is independent of the matrix $\Psi$ under the assumptions that $P(z)$ has a single or double poles at $z=+1$ and a blocking zero at $z=-1$. 
Corollary 15. Let $P(z)$ be a discrete-time, real, rational, proper, negative imaginary system without poles at $z=-1$ but with $P(-1)=0$. Let $Q(z)$ be a discrete-time, real, rational, proper, strictly negative imaginary system. Assume one of the following conditions holds:

i) $\lim _{z \rightarrow 1}(z-1)^{2} P(z)$ is nonsingular;

ii) $\lim _{z \rightarrow 1}(z-1)^{2} P(z)=0$ and $\lim _{z \rightarrow 1}(z-1) P(z)$ is nonsingular.

Then, $[P(z), Q(z)]$ is internally stable if and only if $Q(1)<0$.

Proof. Consider each of the two cases of this corollary separately. Writing $P(z)$ as a Laurent series into either condition 2) or condition 3) of Corollary 14 gives the required result after evaluating the limit and simplifying.

In Subsection 5.1, general stability theorems for discrete-time, real, rational, proper negative imaginary systems including possible poles at $z=+1$ were introduced. We here demonstrate that under the assumption that $P(z)$ has no poles at $z=+1$ and $z=-1$, the general stability results obtained in Theorem 10 and Theorem 11 specialise to the previous stability conditions in Theorem 3 and Theorem 6 . Since it is easy to see that two of the conditions in Theorem 10 (resp. Theorem 11) are trivially equivalent to two of the conditions in Theorem 3 (resp. Theorem 6) by inspection, we only need to show that the remaining inequality in Theorem 10 (resp. Theorem 11) is equivalent to the remaining inequality in Theorem 3 (resp. Theorem 6).

Lemma 16. Let all the assumptions of Theorem 10 hold and furthermore suppose $P(z)$ has no poles at $z=+1$. Then,

$$
\begin{aligned}
& \bar{\lambda}\left[\lim _{z \rightarrow 1}\left[[I-\Psi P(-1)][I-Q(1) P(-1)]^{-1}[Q(1) P(z)-I][I-\Psi P(z)]^{-1}\right]\right]<0 \\
\Leftrightarrow & \bar{\lambda}\left[[I-Q(1) P(-1)]^{-1}(Q(1) P(1)-I)\right]<0 .
\end{aligned}
$$

Proof. Let $M(s)=P((1+s) /(1-s))$ and $N(s)=Q((1+s) /(1-s))$ via the bilinear transformation $z=(1+s) /(1-s)$. Then, the result follows from [30, Lemma 33].

Similarly, if we additionally assume that $P(z)$ has no poles at $z=+1$, the equivalence between the stability conditions in Theorem 11 and Theorem 6 can also be established. 
Lemma 17. Let all the assumptions of Theorem 11 hold and furthermore suppose $P(z)$ has no poles at $z=+1$. Then,

$$
\begin{aligned}
& \bar{\lambda}\left[\lim _{z \rightarrow 1}\left[[I-P(z) \Psi]^{-1}[P(z) Q(-1)-I][I-P(-1) Q(-1)]^{-1}[I-P(-1) \Psi]\right]\right]< \\
& 0 \\
\Leftrightarrow & \bar{\lambda}\left[(P(1) Q(-1)-I)[I-P(-1) Q(-1)]^{-1}\right]<0 .
\end{aligned}
$$

Proof. Let $M(s)=P((1+s) /(1-s))$ and $N(s)=Q((1+s) /(1-s))$ via the bilinear transformation $z=(1+s) /(1-s)$. Then, the result follows from [30, Lemma 34].

\section{Numerical examples}

Two examples are given to illustrate some of the results of this paper.

\subsection{Negative Imaginary without Poles at $z=+1$ and $z=-1$}

The following example is directly taken from [27]. A spring-mass system is given in Figure 2 where two springs $k_{1}$ and $k_{2}$ are used to connect the mass to the wall and masses $m_{1}$ and $m_{2}$ are coupled with a spring $k$. Assume both masses $m_{1}$ and $m_{2}$ are only allowed to move horizontally on a frictionless ground. The forces applied to $m_{1}$ and $m_{2}$ are denoted as $f_{1}$ and $f_{2}$ respectively and the displacements corresponding to these masses are $x_{1}$ and $x_{2}$ respectively. If we set the parameters $k=k_{1}=k_{2}=1 \mathrm{~N} / \mathrm{m}$ and $m_{1}=m_{2}=1 \mathrm{~kg}$ and we discretise the continuous-time signals with a sampling time of $T=2 \mathrm{~s}$, the system with input forces $f_{1}$ and $f_{2}$ and output displacements $x_{1}$ and $x_{2}$ can be modelled (via Newton's second law of motion and a discretisation process) as multi-input multi-output transfer function given by $P(z)=\left[\begin{array}{cc}\frac{3 z^{4}+8 z^{3}+10 z^{2}+8 z+3}{8 z^{4}+8 z^{3}+16 z^{2}+8 z+8} & \frac{z^{4}+4 z^{3}+6 z^{2}+4 z+1}{8 z^{4}+8 z^{3}+16 z^{2}+8 z+8} \\ \frac{z^{4}+4 z^{3}+6 z^{2}+4 z+1}{8 z^{4}+8 z^{3}+16 z^{2}+8 z+8} & \frac{3 z^{4}+8 z^{3}+10 z^{2}+8 z+3}{8 z^{4}+8 z^{3}+16 z^{2}+8 z+8}\end{array}\right]$. We then apply a simple controller $Q(z)=\left[\begin{array}{cc}\frac{-2 z}{3 z+1} & 0 \\ 0 & \frac{-2 z}{3 z+1}\end{array}\right]$. The plant $P(z)$ is a discrete-time, real, rational, proper negative imaginary system without poles at $z=+1$ and $z=-1$ according to Definition 1 and the controller $Q(z)$ is a discretetime, real, rational, proper, strictly negative imaginary system according to Definition 2. [14, Theorem 4.1] is not applicable since $P(-1) Q(-1)=0$ but $Q(-1)=-I_{2} \neq 0$. However, by using Corollary 5 (since $P(-1)=0$ and $\bar{\lambda}[P(1) Q(1)]=-1 / 6<1)$, the closed-loop system is guaranteed to 


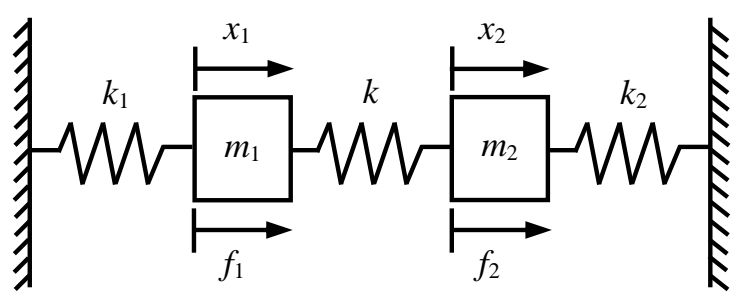

Figure 2: Spring-mass system.

be internally stable with the proposed controller. The closed-loop stability can be confirmed by checking all the poles of the closed-loop transfer function $(I-P(z) Q(z))^{-1} P(z)$. Since all the poles of the closed-loop transfer function $(I-P(z) Q(z))^{-1} P(z)$ are $-0.2308+0.9020 j,-0.2308-0.9020 j$, $-0.5632+0.7925 j,-0.5632-0.7925 j,-0.3023$ and -0.2884 , which are inside the unit circle, the feedback system is internally stable.

\subsection{Negative Imaginary with Poles at $z=+1$}

Let us now consider a positive feedback interconnection as shown in Figure 1 which consists of a discrete-time, real, rational, proper, negative imaginary system $P(z)=\left(3 z^{4}+4 z^{3}+2 z^{2}+4 z+3\right) /\left(2 z^{4}-4 z^{3}+4 z^{2}-4 z+2\right)$ and a discrete-time, real, rational, proper, strictly negative imaginary system $Q(z)=(-3 z+1) / 2 z$. [14, Theorem 4.1] is suitable only for the case where negative imaginary system without poles at $z=+1$ and $z=-1$. In this case, since $P(z)$ is negative imaginary with poles at $z=+1$ according to Definition 1, we cannot apply [14, Theorem 4.1] for stability analysis. Since $P(z)$ is a negative imaginary system with $P(-1)=0$, using condition 2) in Corollary 14, we conclude that the positive feedback interconnection of $P(z)$ and $Q(z)$ is internally stable because we can set $\Psi=Q(1)=-1<0$ to give $\bar{\lambda}\left[\lim _{z \rightarrow 1}\left[[Q(1) P(z)-I][I-\Psi P(z)]^{-1}\right]\right]=$ $\bar{\lambda}\left[\lim _{z \rightarrow 1}\left[[Q(1) P(z)-I][I-Q(1) P(z)]^{-1}\right]\right]=-1<0$. Closed-loop stability can also be confirmed by checking all the poles of the closed-loop transfer function $P(z)(I-Q(z) P(z))^{-1}$. Since these poles are $-0.5803+0.7235 j$, $-0.5803-0.7235 j, 0.3990+0.8830 j, 0.3990-0.8830 j$ and 0.2858 , which are inside the unit circle, the feedback system is internally stable. 


\section{Conclusion}

We proposed feedback stability analysis results for discrete-time negative imaginary systems which are counterparts to the continuous-time results in [30]. These general results can be used to conclude discrete-time closedloop stability for positive feedback interconnections of discrete-time, negative imaginary systems. Examples were also given to illustrate the usefulness of the proposed work.

\section{References}

[1] A. Lanzon and I. R. Petersen, "Stability robustness of a feedback interconnection of systems with negative imaginary frequency response," IEEE Transactions on Automatic Control, vol. 53, no. 4, pp. 1042-1046, May 2008.

[2] M. A. Mabrok, A. G. Kallapur, I. R. Petersen, and A. Lanzon, "Spectral conditions for negative imaginary systems with applications to nanopositioning," IEEE/ASME Transactions on Mechatronics, vol. 19, no. 3, pp. 895-903, Jun. 2014.

[3] B. Bhikkaji, S. O. R. Moheimani, and I. R. Petersen, "A negative imaginary approach to modeling and control of a collocated structure," IEEE/ASME Transactions on Mechatronics, vol. 17, no. 4, pp. 717-727, Aug. 2012.

[4] I. A. Mahmood, S. O. R. Moheimani, and B. Bhikkaji, "A new scanning method for fast atomic force microscopy," IEEE Transactions on Nanotechnology, vol. 10, no. 2, pp. 203-216, Mar. 2011.

[5] A. Sebastian and S. M. Salapaka, "Design methodologies for robust nano-positioning," IEEE Transactions on Control Systems Technology, vol. 13, no. 6, pp. 868-876, Nov. 2005.

[6] J. Wang, A. Lanzon, and I. R. Petersen, "Robust output feedback consensus for networked negative-imaginary systems," IEEE Transactions on Automatic Control, vol. 60, no. 9, pp. 2547-2552, Sep. 2015.

[7] — , "Robust cooperative control of multiple heterogeneous negativeimaginary systems," Automatica, vol. 61, pp. 64-72, Nov. 2015. 
[8] I. R. Petersen and A. Lanzon, "Feedback control of negative-imaginary systems," IEEE Control Systems Magazine, vol. 30, no. 5, pp. 54-72, Oct. 2010 .

[9] J. L. Fanson and T. K. Caughey, "Positive position feedback control for large space structures," AIAA Journal, vol. 28, no. 4, pp. 717-724, Apr. 1990 .

[10] I. R. Petersen, "Negative imaginary systems theory and applications," Annual Reviews in Control, vol. 42, pp. 309-318, 2016.

[11] C. Cai and G. Hagen, "Stability analysis for a string of coupled stable subsystems with negative imaginary frequency response," IEEE Transactions on Automatic Control, vol. 55, no. 8, pp. 1958-1963, Aug. 2010.

[12] A. van der Schaft, "Positive feedback interconnection of Hamiltonian systems," in Proceedings of the 50th IEEE Conference on Decision and Control and European Control Conference, Orlando, FL, USA, Dec. 2011, pp. 6510-6515.

[13] A. Ferrante, A. Lanzon, and L. Ntogramatzidis, "Foundations of not necessarily rational negative imaginary systems theory: Relations between classes of negative imaginary and positive real systems," IEEE Transactions on Automatic Control, vol. 61, no. 10, pp. 3052-3057, Oct. 2016.

[14] — - "Discrete-time negative imaginary systems," Automatica, vol. 79, pp. 1-10, May 2017.

[15] A. Ferrante and L. Ntogramatzidis, "Some new results in the theory of negative imaginary systems with symmetric transfer matrix function," Automatica, vol. 49, pp. 2138-2144, 2013.

[16] — , "On the definition of negative imaginary system for not necessarily rational symmetric transfer functions," in European Control Conference, Zurich, Switzerland, 2013, pp. 312-316.

[17] M. Liu and J. Xiong, "On non-proper negative imaginary systems," Systems and Control Letters, vol. 88, pp. 47-53, Feb. 2016. 
[18] M. R. Opmeer, "Infinite-dimensional negative imaginary systems," IEEE Transactions on Automatic Control, vol. 56, no. 12, pp. 29732976, Dec. 2011.

[19] J. Xiong, A. Lanzon, and I. R. Petersen, "Negative imaginary lemmas for descriptor systems," IEEE Transactions on Automatic Control, vol. 61, no. 2, pp. 491-496, Feb. 2016.

[20] A. Lanzon, Z. Song, S. Patra, and I. R. Petersen, "A strongly strict negative-imaginary lemma for non-minimal linear systems," Communications in Information and Systems, vol. 11, no. 2, pp. 139-152, 2011.

[21] Z. Song, A. Lanzon, S. Patra, and I. R. Petersen, "Towards controller synthesis for systems with negative imaginary frequency response," IEEE Transactions on Automatic Control, vol. 55, no. 6, pp. 1506-1511, Jun. 2010.

[22] — - "Robust performance analysis for uncertain negative-imaginary systems," International Journal of Robust and Nonlinear Control, vol. 22, no. 3, pp. 262-281, Feb. 2012.

[23] — - "A negative-imaginary lemma without minimality assumptions and robust state-feedback synthesis for uncertain negative-imaginary systems," Systems and Control Letters, vol. 61, no. 12, pp. 1269-1276, Dec. 2012.

[24] J. Xiong, J. Lam, and I. R. Petersen, "Output feedback negative imaginary synthesis under structural constraints," Automatica, vol. 71, pp. 222-228, Sep. 2016.

[25] B. C. Levy and M. Zorzi, "A contraction analysis of the convergence of risk-sensitive filters," SIAM Journal on Control and Optimization, vol. 54, pp. 2154-2173, 2016.

[26] M. Zorzi, "Robust kalman filtering under model perturbations," IEEE Transactions on Automatic Control, vol. 62, pp. 2902-2907, 2017.

[27] J. Xiong, I. R. Petersen, and A. Lanzon, "A negative imaginary lemma and the stability of interconnections of linear negative imaginary systems," IEEE Transactions on Automatic Control, vol. 55, no. 10, pp. 2342-2347, Oct. 2010. 
[28] M. A. Mabrok, A. G. Kallapur, I. R. Petersen, and A. Lanzon, "Generalizing negative imaginary systems theory to include free body dynamics: Control of highly resonant structures with free body motion," IEEE Transactions on Automatic Control, vol. 59, no. 10, pp. 2692-2707, Oct. 2014.

[29] S. Z. Khong, I. R. Petersen, and A. Rantzer, "Robust feedback stability of negative imaginary systems: An integral quadratic constraint approach," in Proceedings of the 14th European Control Conference, Linz, Austria, Jul. 2015.

[30] A. Lanzon and H. J. Chen, "Feedback stability of negative imaginary systems," IEEE Transactions on Automatic Control, vol. 62, no. 11, pp. 5620-5633, Mar. 2017.

[31] M. Liu and J. Xiong, "Connections between discrete- and continuoustime results for positive real and negative imaginary systems," in IEEE 55th Conference on Decision and Control, Las Vegas, USA, Dec. 2016, pp. 4931-4936. 